\title{
Lidil
}

Revue de linguistique et de didactique des langues

$64 \mid 2021$

Le passif dans la langue parlée

Jean-Louis Chiss et Jacques David, Didactique du français. Enjeux disciplinaires et étude de la langue Malakoff, Armand Colin, «Collection U », 2018, 349 p.

\section{Beatriz Villa}

\section{OpenEdition}

Journals

Édition électronique

URL : https://journals.openedition.org/lidil/9323

DOI : 10.4000/lidil.9323

ISSN : 1960-6052

Éditeur

UGA Éditions/Université Grenoble Alpes

Édition imprimée

ISBN : 978-2-37747-315-1

ISSN : $1146-6480$

Référence électronique

Beatriz Villa, « Jean-Louis Chiss et Jacques David, Didactique du français. Enjeux disciplinaires et étude de la langue », Lidil [En ligne], 64 | 2021, mis en ligne le 01 novembre 2021, consulté le 26 novembre 2021. URL : http://journals.openedition.org/lidil/9323 ; DOI : https://doi.org/10.4000/lidil.9323

Ce document a été généré automatiquement le 26 novembre 2021.

(c) Lidil 


\title{
Jean-Louis Chiss et Jacques David, Didactique du français. Enjeux disciplinaires et étude de la langue
}

\author{
Malakoff, Armand Colin, « Collection U », 2018, 349 p.
}

\section{Beatriz Villa}

\section{RÉFÉRENCE}

Jean-Louis Chiss et Jacques David, Didactique du français. Enjeux disciplinaires et étude de la langue, Malakoff, Armand Colin, «Collection U », 2018, 349 p.

1 L'ouvrage rassemble et enrichit une série d'articles intitulée « Didactique du français et étude de la langue", signés par les mêmes auteurs dans la collection Le français aujourd'hui en 2012. Cette reconfiguration se veut ouverte à un large public, bien que la lecture soit dense et technique. L'ouvrage présente des éléments historiques, épistémologiques et pratiques sur la didactique de la grammaire et de l'orthographe. Les auteurs s'appuient sur la vivacité des débats éducatifs et politiques que l'enseignement du français suscite au sein de la société, ce qui justifie la finesse de leur démarche analytique structurée en trois parties.

2 Ils étudient d'abord les grammaires de référence de plusieurs pays francophones, afin de savoir si le modèle français de manuel grammatical est dominant, et si les variations locales de la langue et la formation des enseignants sont prises en compte. Une deuxième partie est consacrée au domaine de l'enseignement de la grammaire, interrogeant les conditions des cursus de formation des enseignants de français quant aux spécificités didactiques de la discipline, l'utilité de l'étude de la langue dans les classes et les contenus grammaticaux à enseigner. La dernière partie aborde les questions et les polémiques récentes de l'enseignement de l'orthographe et propose des approches qui, quoique renouvelées, reconnaissent «la nécessité sociale et communicationnelle de l'orthographe » (p. 227). 
3 Les discours de crise portant à la fois sur la langue française et sur son enseignement avancent des critiques qui se réduisent à l'utilisation d'un « jargon» (p. 49). Les auteurs citent entre autres: Fumaroli (2003, 2005), un "pédagogisme prétentieux", une "pression rhétorique ", un "caractère super-élitiste "; Grange et Leroux (2005), des "savoirs savants de la linguistique»; Debray (2005), des "linguisteries tristes et compliquées »; Brisset (2006), un « stupéfiant jargon ». Ils rappellent cependant deux piliers de la construction disciplinaire. D'une part, les élèves ont besoin de développer une terminologie spécifique pour être capables de raisonner et de parler du fonctionnement de la langue. D'autre part, au-delà d'un problème de surface comme celui des nomenclatures, la question de fond consiste à repenser des savoirs grammaticaux plus efficaces.

4 Les enseignants de français, en FLM et en FLE, se réjouiront de trouver dans cet ouvrage certains contenus grammaticaux traditionnellement enseignés, qu'il serait temps de déconstruire. C'est le cas par exemple des trois groupes de la conjugaison verbale, que les auteurs proposent de remplacer par les bases morphologiques des verbes (p. 56 et 130). Il en va de même pour les compléments circonstanciels qui ne devraient plus être classés dans un même et seul ensemble, mais selon leur fonction syntaxique (p. 55). Il s'agirait de faire valoir les liens entre syntaxe et sémantique, plutôt que de retenir la classique analyse par fonctions (sujet, complément d'objet et complément circonstanciel). En effet, celle-ci peut s'avérer fausse lorsqu'il y a recours à des opérations de suppression ou de déplacement. Les auteurs proposent de nombreux exemples pour illustrer ces nécessaires évolutions aussi bien au niveau de la terminologie que de la logique de la progression des contenus grammaticaux.

5 Les grammaires de référence analysées dans l'ouvrage (Genevay (1994) pour la Suisse, Chartrand et coll. (1999-2011) pour le Québec, Wilmet (1997-2010) pour la Belgique, et Riegel, Pellat \& Rioul (1994-2004) pour la France) ne développent pas de perspective sociolinguistique. Les auteurs expliquent cette absence de contenu variationnel par la difficulté de systématisation et donc de délimitation de l'objet à enseigner. En somme, ces grammaires cherchent à répondre aux exigences de standardisation, voire de «simplification» (p. 93) des connaissances que présuppose l'école. Autrement dit, la prise en compte des variations sociolinguistiques (sociales, géographiques, situationnelles ou temporelles) génère des changements sur l'objet, les corpus et les méthodologies d'enseignement. Selon les auteurs, il faudrait concevoir des grammaires qui fassent dialoguer le système et ses variations, et ils interrogent l'impact de ces deux entrées sur la relation à l'autre selon qu'il s'agisse d'une langue première ou d'une langue étrangère.

6 L'exemple de la lecture met en évidence la nécessité de cohérence entre continuité et spécificité de la compétence de lecture dans les programmes scolaires. Au collège, le temps de lecture en classe est réduit, alors que les apprenants sont censés lire de plus en plus en autonomie. Il pourrait donc paraitre normal que les enseignants trouvent que les enfants ne savent pas lire. De la même manière, la connaissance limitée de la grammaire, la baisse du niveau, la mauvaise orthographe sont récurrentes dans les débats. Ce que Chiss et David rappellent dans cet ouvrage est que l'étude de la langue n'a rien d'évident ni d'universel, et que comme les mathématiques ou les sciences de la vie, cette discipline développe des savoirs spécifiques. Il ne s'agit pas seulement d'améliorer les pratiques dans la langue, mais aussi de construire des connaissances sur la langue. Les enjeux majeurs de la constitution disciplinaire sont la transposition 
didactique et la délimitation de l'objet: «L'activité grammaticale doit-elle aboutir à l'explication d'un savoir sur la langue ou se contenter de manipulations ordonnées sans emploi d'une terminologie détaillée et fixée ?» (p. 106)

7 L'enseignement de la langue doit résulter d'une articulation entre l'institution scolaire, la recherche en linguistique et en didactique, les contenus et la formation des enseignants. Ces derniers exercent leur métier comme des "spécialistes de tout» (p. 98) : littérature, culture, grammaire, orthographe, lexique, enseignement de l'oral, de l'écrit, de la lecture, etc. Tant que cette convergence ne sera pas possible, les enseignants resteront dans le flou de leur discipline. Car à l'impératif de devoir choisir des priorités, s'ajoutent des hétérogénéités d'ordre social, culturel, cognitif et langagier. C'est dans ce sens que l'ouvrage met au centre la recherche et la formation des enseignants, à partir de perspectives diverses (historiques, linguistiques, psycholinguistiques, culturelles et méthodologiques).

8 La lecture de cet ouvrage saura intéresser les chercheurs en linguistique et en didactique des langues, tout autant que les décideurs des programmes scolaires de français langue maternelle, sur les questions de nomenclature grammaticale et de progression. Les enseignants en exercice et en formation trouveront des outils et des démarches d'analyse linguistique (phrase/texte-genre textuel/discours) pour aider les apprenants à développer un métalangage, mieux comprendre leur langue et ainsi, devenir plus performants.

\section{AUTEURS}

\section{BEATRIZ VILLA}

Univ. Grenoble Alpes, LIDILEM, 38000 Grenoble, France 